\title{
Structural and Electron-hopping Studies of Pr and Nd substituted La2/3Ba1/3MnO3 Manganites
}

\begin{abstract}
The significance of substituting concentrations of $\mathrm{Pr}$ and $\mathrm{Nd}$ at La-sites, in $\mathrm{La} 0.67 \mathrm{Ba} 0.33 \mathrm{MnO} 3$ perovskite compounds, for the structural and electrical properties have been studied. Polycrystalline samples (La1-x Prx)0.67Ba0.33MnO3 and (La1-x $\mathrm{Ndx}) 0.67 \mathrm{Ba0} .33 \mathrm{MnO} 3$ with $\mathrm{x}=0.00,0.167,0.333,0.5,0.667,0.833$ and 1.0 were synthesized via conventional solid-state reaction in the bulk. All of the samples were calcined at $900 \mathrm{oC}$ for 12 hours, pelletized and sintered at $1300 \mathrm{oC}$ for 24 hours and investigated. In this paper the structural patterns and microstructural properties of bulk samples have been investigated via X-ray diffractometry (XRD) and scanning electron microscopy (SEM). XRD patterns show that these systems are single-phase, with orthorhombic distorted perovskite structures. The electrical property, Tp, was determined by using standard four-point probe resistivity measurements in the temperature range of $20 \mathrm{~K}$ to $300 \mathrm{~K}$. The result shows that $\mathrm{Pr}$ and $\mathrm{Nd}$ dopants shift the value of TP to a lower temperature. When the temperature is above $\mathrm{Tp}, \mathrm{T}>\mathrm{Tp}$, the variation of the electrical resistance was found to follow the an Arhenius-type law, $\rho=\rho o \exp (-\mathrm{Ea} / \mathrm{KBT})$. It was used to calculate the activation energy of every sample. The resistivity curves show semiconducting behavior of all samples above their Tp.
\end{abstract}

Keyword: Arrhenius-Type Law Activation Energy (Ea); Meta-Insulator Transistor Temperature (Tp); Resistivity ( $\rho$ ) 